\title{
Implementation of Short- and Medium-Term Earthquake Forecasts
}

\author{
Rodolfo Console, ${ }^{1}$ Koshun Yamaoka, $^{2}$ and Jiancang Zhuang ${ }^{3}$ \\ ${ }^{1}$ Istituto Nazionale di Geofisica e Vulcanologia, 00143 Roma, Italy \\ ${ }^{2}$ Graduate School of Environmental Studies, Nagoya University, Nagoya 464-8601, Japan \\ ${ }^{3}$ Institute of Statistical Mathematics, Tokyo 106-8569, Japan
}

Correspondence should be addressed to Rodolfo Console, console@ingv.it

Received 11 March 2012; Accepted 11 March 2012

Copyright ( $\odot 2012$ Rodolfo Console et al. This is an open access article distributed under the Creative Commons Attribution License, which permits unrestricted use, distribution, and reproduction in any medium, provided the original work is properly cited.

The cases of the recent destructive earthquakes that occurred with impressive frequency in Sichuan (China, 2008), Italy (2009), Haiti (2010), Chile (2010), New Zealand (2010), and Tohoku (Japan, 2011) have shown that, in present state, scientific researchers have achieved little or almost nothing in the implementation of short- and medium-term earthquake prediction, which would be useful for disaster mitigation measures.

This regrettable situation could be ascribed to the present poor level of achievements in earthquake forecast. In fact, although many methods have been claimed to be capable of predicting earthquakes (as numerous presentations on earthquake precursors regularly show at every international meeting), the problem of formulating such predictions in a quantitative, rigorous, and repeatable way is still open.

In the last decade some short- and intermediate-term forecasting models have been proposed in a way that can provide us with the estimate of earthquake occurrence probability in a specific window of their origin times, epicentral coordinates, and magnitudes. They have been rigorously tested in both prospective and retrospective ways against observed seismic activity. However, it seems that these applications are still far away from our practical purposes (see [1], for a comprehensive review of the progress achieved in the last ten years on seismicity-based earthquake forecasts).

On the other hand, another problem of practical implementation of earthquake forecasting could be due to the lack of common understanding and exchange of information between the scientific community and the governmental authorities that are responsible for earthquake damage mitigation in each country: they operate in two different environments, they aim at different tasks, and they generally speak two different languages. In particular, the way how seismologists should formulate their forecasts and how they should transfer them to decision-makers and to the public is still a tricky issue. The case that seven scientists belonging to the Committee on Seismic Risk of the Italian Civil Protection Agency were legally prosecuted after the May 5th, 2009, L'Aquila earthquake is a clamorous example of this kind of problems [2]

It is clear that the formulation of probabilistic earthquake forecasts with large uncertainties in space and time and very low probability levels is still difficult to be used by decisionmaking people. In real circumstances the authorities deal with critical problems related to the high cost of evacuating the population from an area where the scientific methods estimate an expected rate of destructive earthquake as one in many thousand days, while they require much more deterministic statements. A new name, "operational earthquake forecasting," has been given to the process of providing usable information on future earthquakes or ongoing seismic sequences. An extensive report on the potential of such a process has been written by the International Committee appointed by the Italian Civil Protection Agency [3].

In this special issue, we were aiming to assess the status of the art of earthquake forecasts and their applicability. Therefore, we invited authors to report methods and case 
studies that could concretely contribute or, at least seemed promising, to improve the present frustrating situation, regarding the practical use of earthquake forecasts. Seven contributions out of the 22 submissions to this special issue were accepted.

Two contributions present reviews of the present status of earthquake forecasting and its validation. One of them, titled "Precursor-like anomalies prior to the 2008 Wenchuan Earthquake: a critical-but-constructive review" by T. Ma and $\mathrm{Z}$. Wu, is a critical review of more than 200 papers reporting the retrospective analysis of precursory anomalies observed prior to the 2008 Wenchuan earthquake in China. The material collected and used for the analysis contains a wide range of precursor-like anomalies observed at different time scales. These anomalies include, among others, seismicity changes, deformation measurements, strain/stress measurements, physical variations, gravity recordings, ionospheric anomalies, and geothermal and atmospheric anomalies. Some of the observations show consistent characteristic times, such as 2-4 days and 1-2 years, which may reveal the preparation and approaching process of this great inland earthquake, and suggest the need for further investigation. A more quantitative approach in statistical sense is considered in "Evaluating the RELM test results" by M. K. Sachs et al. They start from the results of the Regional Earthquake Likelihood Models (RELM) test for earthquake forecasting. Five different forecasting methods were considered in the test. In particular, the authors utilize a methodology developed in the atmospheric sciences, specifically for tornadoes, to compare the forecast results. The best forecasts are about one order of information significance better than random forecasts. The paper also includes a discussion on alternative methods of evaluation of the performance of RELM forecasts and on the relative merits of alarm-based versus probabilitybased forecasts.

Two papers report case studies on different kinds of earthquake precursors. One of them, titled "Spatiotemporal relationship between geodetic and seismic quantities: a possible clue to preparatory processes of $M \geq 6.0$ inland earthquakes in Japan," is by Kawamura et al. They aimed to formulate a model to characterize the physical conditions associated with large earthquake occurrence. In their study, they focused on assessing the relationship between geodetic and seismic quantities and attempted to find the pair of related quantities that most likely indicates preparatory processes of large earthquakes in Japan. Their study revealed that the pair of absolute dilatation rate and seismic energy shows the highest statistical performance. The second paper of this group is titled "Medium-term earthquake forecast using gravity monitoring data: evidences from the Yutian and Wenchuan earthquakes in China, by Y. Zhu and F. B. Zhan. They review the gravity monitoring data and methods that had allowed a medium-term forecast of the Yutian and Wenchuan, 2008 earthquakes in China. This paper suggests that gravity changes derived from regional gravity monitoring data could potentially be a useful medium-term precursor of large earthquake, but significant additional research is needed to evaluate and validate this hypothesis.
Other two papers deal with ongoing research projects on earthquake precursors aiming at the development of physical models. One of these is "The new avalanche-like stochastic model for parameterization of seismicity and its application to the South Sakhalin Island seismicity," by M. V Rodkin and I. Tikhonov. In this paper, the seismic process is regarded as an assemblage of randomly developing episodes of avalanchelike relaxation. Such a model is considered as an alternative to the model based on the concept of SOC (self-organized criticality). An advantage of this approach consists in a clear physical meaning of the model parameters. An anomalous increase of the parameter called "metastability" was found in connection with two earthquakes occurring in the southern Sakhalin Island in 2006 and 2007, which indicates an increase in probability of the occurrence of a future earthquake. The next paper, with the ambitious title of "A deterministic approach to earthquake prediction," by V. Sgrigna and L. Conti, addresses the importance of combining ground and space observations of earthquake precursors. The authors describe a few projects and experiments carried out with the aim of providing support to the theoretical interpretation of seismogenic processes.

The final contribution, "Probabilistic seismic hazard analysis for Yemen," by R. Mohindra et al. is a typical example of probabilistic seismic hazard assessment (PSHA) based on the recognition of the principal seismic sources in and around the territory of Yemen. It was recognized that the largest contribution to the PSHA comes from the West Arabian Shield seismic zone.

\section{Acknowledgment}

We thank many anonymous reviewers for their careful reviews and constructive suggestions in improving each submission.

\section{Rodolfo Console Koshun Yamaoka Jiancang Zhuang}

\section{References}

[1] K. F. Tiampo and R. Shcherbakov, "Seismicity-based earthquake forecasting techniques: ten years of progress," Tectonophysics, vol. 522-523, pp. 89-121, 2012.

[2] N. Nosengo, "Italy puts seismology in the dock," Nature, vol. 465, no. 7301, article 992, 2010.

[3] T. H. Jordan, Y.-T. Chen, P. Gasparini et al., "Operational earthquake forecasting. State of knowledge and guidelines for utilization," Annals of Geophysics, vol. 54, article 4, 2011. 

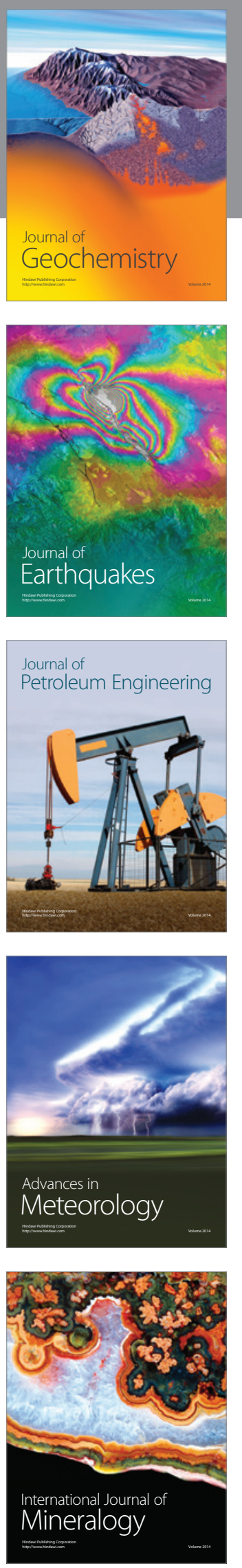
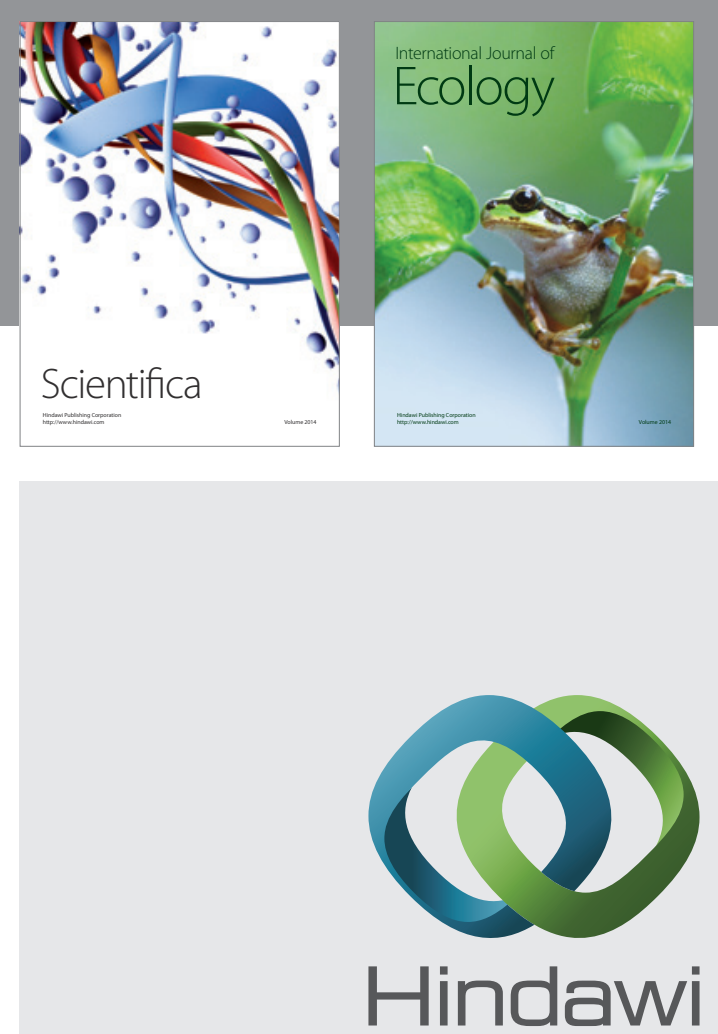

Submit your manuscripts at http://www.hindawi.com
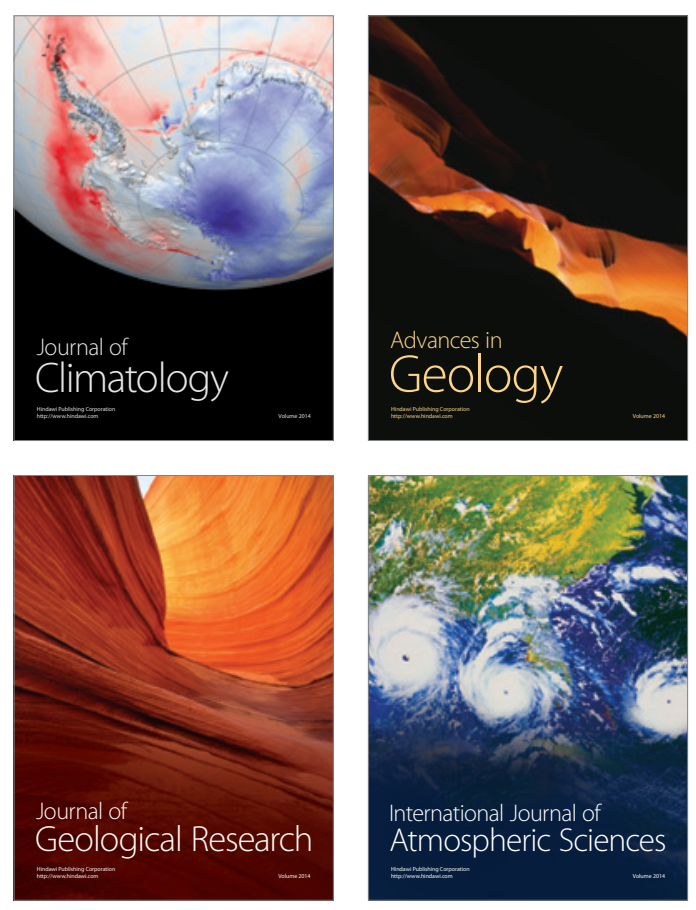
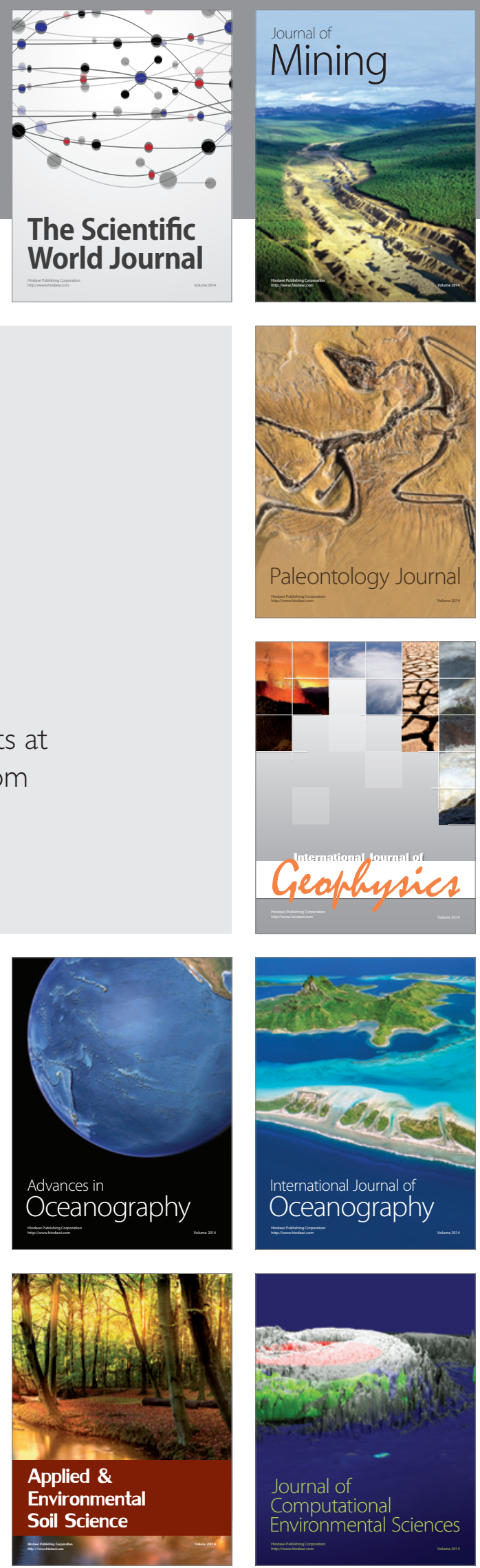